stressful environment for patients. All medical and non-medical staff were trained on LGBTQ sexual health issues and some on Club Drug related issues.

Aim To assess patient satisfaction with the new service, a questionnaire was undertaken seven months after service commencement.

Methods Over a four-week period, questionnaires were handed to patients by reception at booking. Thirty-six questionnaires were returned.

Results Patients rated the service as excellent $81 \%$ (26/32) very good 14\% (5/32) or good 3\% (1/32). All patients who answered said they would attend again $100 \%(32 / 32)$. Most would recommend it to a friend $97 \%(32 / 33)$. Patient staff satisfaction was high, with 96\% (29/30) stating they felt listened to. Patients felt treated with respect and dignity always 97\% (31/32) or sometimes $3 \%(1 / 32) .83 \%(30 / 36)$ of patients attended specifically with LGBTQ concerns, of these 93\% (28/30) felt confident in discussing concerns with staff, $7 \%(2 / 30)$ to some extent. $77 \%$ (24/31) indicated that drugs or alcohol were related to their visit. Only $21 \%(5 / 24)$ felt they could raise this topic with staff and $79 \%(19 / 24)$ stated they couldn't.

Discussion Staff training on managing vulnerable clients' health needs enables confidant and approachable health care. This provides patients with opportunities to verbalise health anxieties, facilitating opportunistic healthcare interventions. Drug and Alcohol training for all staff will enhance the patient care package.

\section{P228 EVALUATING THE USE OF EXTRA-GENITAL CHLAMYDIA TRACHOMATIS (CT) AND NEISSERIA GONORRHOEA (GC) NUCLEIC ACID AMPLIFICATION TESTS (NAATS) IN NON- MSM POPULATIONS}

Maria Elbadawi*, Rosemary Lockyer, Amy Bennett. Ashford and St Peter's Hospitals NHS Foundation Trust, Chertsey, UK

\subsection{6/sextrans-2016-052718.276}

Background/introduction Our clinic performs routine oral and anal CT and GC NAATs following disclosure during sexual history of oral or receptive anal sex. Specimens are analysed using the Abbott M2000 assay, costing $£ 4.50$ per dual NAAT.

$\operatorname{Aim}(\mathrm{s}) /$ objectives We report the findings of this practice, focusing on clinical and partner notification outcomes, and the cost per diagnosis.

Methods We included all non-MSM patients with isolated extragenital CT or GC infection from $1 / 8 / 14$ to $31 / 7 / 15$. We excluded contacts of either infection, plus those without GC confirmed by culture or second NAAT platform. Retrospective case note review was performed for identified cases.

Results In total 8796 NAATs were processed in non-MSM patients, of which 3813 (43\%) were extra-genital. In heterosexual men we identified 3 pharyngeal GC cases and one contact, and one pharyngeal CT case with one contact. In women there were 2 pharyngeal GC cases, one pharyngeal CT case and one rectal CT case with no additional contacts. The spend per pharyngeal diagnosis was $£ 1519$ in males and $£ 3138$ in women. The spend per rectal diagnosis in women was $£ 1665$.

Discussion/conclusion Our screening practice has picked up only a small number of cases which would otherwise have been missed. $25 \%(2 / 8)$ of those cases had verified contacts identified. The cost implication of such screening is high, meaning that ongoing routine screening is not viable. In our relatively low- prevalence setting we now intend to target our screening in line with BASHH CEG guidance which will provide significant cost savings.

\section{P229 CLINICIAN AND COMMUNITY COLLABORATION ON PREP IN THE UK - A NARRATIVE}

${ }^{1}$ Mags Portman*, ${ }^{2}$ GrEG Owen, ${ }^{3}$ Killian Quinn, ${ }^{2}$ Alex Craddock, ${ }^{8}$ Marc Thompson, ${ }^{4}$ Nneka Nwokolo, ${ }^{5}$ lain Reeves, ${ }^{6}$ Jake Bayley, ${ }^{7}$ Michael Brady, ${ }^{8}$ Will Nutland, ${ }^{4,9}$ Sheena McCormack. 'Mortimer Market Centre, Central and North West London NHS Foundation Trust, London, UK; ${ }^{2}$ WantPrEPNow.co.uk, London, UKi ${ }^{3}$ King's College Hospital NHS Foundation Trust, London, UKi ${ }^{4} 56$ Dean Street, Chelsea and Westminster Hospital NHS Foundation Trust, London, UK; ${ }^{5}$ Homerton University Hospital NHS Foundation Trust, London, UK; ${ }^{6}$ Barts Health NHS Trust, London, UK; ${ }^{7}$ Terence Higgins Trust, London, UK; ${ }^{8}$ Prepster.info, London, UK; ${ }^{9}$ Medical Research Unit Clinical Trials Unit at UCL London, UK

\subsection{6/sextrans-2016-052718.277}

Background The PROUD study showed that PrEP can be successfully provided via sexual health clinics in the UK. The delay in widespread availability of PrEP has seen renewed grassroots activism from the community sector.

Methods We document the timeline and benefits of a close relationship between community activists and clinicians in advancing the PrEP agenda.

Results Community engagement during the PROUD trial led on to increased advocacy and activism from October 2014 when interim results were published. Launch of the websites IWantPrEPNow.co.uk (with a click to buy generic PrEP) and PrEPster. info in October 2015 further galvanised clinicians and community advocates into greater joined up action. The benefits of this included: 1) Mutual social media support; advancing the PrEP agenda amongst potential PrEP users and clinicians, 2) Obtaining GMC input on providing clinical advice regarding generics, 3) Pushing for free monitoring for current generic PrEP users, 4) Sharing of therapeutic drug monitoring data, 5) Community input into staff training and service development such as production and review of patient information, and 6) Two way learning; sharing PrEP users perspective and evidence based research. Discussion/conclusion The lack of availability of PrEP in the UK is proving a challenge to both community advocates and clinicians. We wish to celebrate the achievements made within the confines of the current political climate, in particular working to ensure patient safety. There is a history of successful clinician/ community collaboration in GUM/HIV medicine; with an NHS England PrEP roll out we need to reflect this to drive uptake. If NHS England decides against PrEP funding, we suggest that continued collaboration, support and innovation will be paramount.

\section{P230 REVIEW OF SYPHILIS MANAGEMENT AT A BUSY URBAN STI CLINIC}

Niamh Lynn*, Padraig Calpin, Brendan O'Kelly, Ksenia Davenport, Fiona Lyons. GUIDe Clinic, St. James's Hospital, Dublin, Ireland

\subsection{6/sextrans-2016-052718.278}

Background/introduction Appropriate management of syphilis is vital in order to avoid late complications. A retrospective cohort study of patients with positive serology at an urban STI clinic where the BASHH guidelines are followed was undertaken. 
Aim(s)/objectives Evaluate:: Documentation of syphilis stage, Treatment, Interval between treatment and first follow-up RPR, RPR 12 months post treatment -determine those with negative RPR; fourfold reduction in RPR or documented as "serofast".

Methods A list of positive serology between April 2013 and March 2014 was generated. Patients managed at clinic were identified and their electronic record reviewed. Descriptive column statistics were used for data analysis.

Results 93 cases of syphilis were managed. Median age 35 years (range 20-74); 82 (88\%) cases male; 47 (51\%) HIV positive. Stage was documented in $45(48 \%)$. Of those with undocumented stage, RPR $>/=1: 8$ in 29,25 were treated with single benzathine-penicillin. 85 received penicillin- based treatment, 48 (52\%) received single dose benzathine penicillin. 8 received doxycycline. Median interval from treatment to subsequent RPR 40 days (range14- 219). 12 months post treatment, 48 (52\%) had negative or "neat" RPR. $58(62 \%)$ a fourfold reduction. In those with a positive RPR, 4 were deemed to be "serofast".

Discussion/conclusion Syphilis continues to be a common infection in men. The majority of patients were treated with penicillin as per BASHH guidelines. There is room for improvement in documenting stage of infection and outcome in those who fail to have a fourfold reduction in RPR at 12 months.

\section{P231 SERVICE IMPROVEMENT REVIEW OF SEXUAL HEALTH SERVICE DNA RATES}

Naomi Kaufman*, Claire Dewsnap, Lauren Theaker. Sheffield Teaching Hospital Foundation Trust, Sheffield, UK

\subsection{6/sextrans-2016-052718.279}

Background/introduction Reductions in sexual health budgets have occurred across England. There is significant focus on the Public Health Outcomes framework and requirements to deliver improved services for less. Sexual health services are examining ways to be more efficient. In 2015 we set out to examine aspects of inefficiency within our service. We highlighted a high 'Did Not Attend' (DNA) rate for Follow up patients as a significant inefficiency. We describe a service review undertaken as part of service improvement plan looking at the reasons and triggers for patients not attending or cancelling their appointments.

Aim(s)/objectives To describe associated factors with not attending appointments when a follow up (FU) appointment has been arranged. To identify solutions to reduce DNA rates to release extra capacity for new appointments.

Methods Focused initial data collection on Doctor follow up stream for November 2015. Aimed to look at whether the following impacted on DNA rate: SMS reminders, Time between booking \& appointment and Reason for follow up

Results The overall DNA rate for Doctor Follow up appointments is $23.7 \%$. Median time from appointment made to appointment either attended or not was the same, 18 days. Patients re-attending for Gonorrhoea and chlamydia treatment, Post exposure Prophylaxis FU, Herpes FU and patients with ongoing symptoms attended well. Patients attending for hepatitis $\mathrm{B}$ vaccination, window period testing, FU for Pelvic inflammatory disease and Chlamydia Test of cure were significantly more likely to DNA. A survey was conducted to ask patients what were the acceptable means of avoiding DNAs.

Discussion/conclusion A total of 165 appointments were missed in November 2015, equivalent to 10 appointments every day. Introducing additional text reminders, using social influence models to increase patient cancellation, were all likely to reduce DNA rate and increase capacity for new appointments.

\section{P232 KEEPING ON TOP OF BV - AN AUDIT OF BACTERIAL VAGINOSIS AND STI SCREENING IN GUM CLINIC ATTENDEES REQUESTING A TERMINATION OF PREGNANCY}

Elspeth Bird*, Christina Micanovic, Catherine McCrann, Aseel Hegazi. St Georges Hospital, London, UK

\subsection{6/sextrans-2016-052718.280}

Background/introduction BASHH guidelines currently recommend universal testing for all GUM clinic attendees and all those undergoing termination of pregnancy (TOP). Screening for and treating bacterial vaginosis (BV) is recommended by BASHH in order to reduce the incidence of post TOP endometritis and PID.

Aim(s)/objectives To audit whether STI screening, to include testing for BV, is being carried out in patients attending a GUM service requesting TOP referral.

Methods Retrospective case note review of 100 consecutive patients seen requesting TOP referral in an Inner London GUM clinic $1 / 1 / 15-1 / 2 / 15$.

Results Case notes of 100 were reviewed. Median age was $25 \mathrm{yrs}$ (range $16-49$ yrs). $21 \%$ of patients $(n=21)$ were of Black ethnicity. 28/50 (56\%) reported previous pregnancy and 19/43 (44\%) previous TOP. Sexual histories were documented in 53\% $(n=53)$ of cases. $43 \%(43 / 100)$ of patients were documented to be asymptomatic. $38 / 100$ patients had an STI screen and of the remainder $37 \%$ were documented to have declined. STI screening included serology testing for HIV/STS in $68 \%$ $(n=26)$ of cases and microscopy for BV/TV in $42 \%(n=16)$. No asymptomatic patients were screened for BV. STI prevalence in those screened was BV 50\% (8/16), Chlamydia 8\% (3/37), Gonorrhoea 5\% (2/37).

Discussion/conclusion Adherence to BASHH guidelines was inconsistent particularly in relation to STI screening and BV screening in asymptomatic women seeking TOP. STI prevalence was relatively high in those screened. A designated clinical proforma may improve documentation and testing rates.

\section{P233 A CASE OF CMV (CANCER MIMICKING VIRULENCE)}

'Johnny Boylan*, ${ }^{1}$ Peter Greenhouse, ${ }^{1,2}$ Patrick Horner. ${ }^{1}$ Bristol Sexual Health Centre, Bristol, UK; ${ }^{2}$ University of Bristol School of Social and Community Medicine, Bristol, UK

\subsection{6/sextrans-2016-052718.281}

Background/introduction Generalised lymphadenopathy and fatigue have a broad differential diagnosis ranging from curable infections to lymphoma. Certain diagnoses can be delayed or missed altogether when patients do not present to sexual health clinics.

Aim(s)/objectives Highlight secondary syphilis as a cause of lymphadenopathy amongst non-sexual health colleagues. Expand syphilis testing beyond sexual health clinics, even in the absence of classical risk factors.

Methods Case report of secondary syphilis which was misdiagnosed despite numerous investigations in primary and secondary care. 